Check for updates

Cite this: Phys. Chem. Chem. Phys., 2017, 19, 23146

Received 13th June 2017, Accepted 7th August 2017 DOI: $10.1039 / c 7 c p 03949 b$ rsc.li/pccp

\section{Helium-3 gas self-diffusion in a nematically ordered aerogel at low temperatures: enhanced role of adsorption}

\author{
Vyacheslav Kuzmin, (D) *a Kajum Safiullin, (D) *ab Andrey Stanislavovas ${ }^{a}$ and \\ Murat Tagirov (D) ab
}

\begin{abstract}
We performed ${ }^{3} \mathrm{He}$ gas diffusion measurements for the first time in a highly porous ordered $\mathrm{Al}_{2} \mathrm{O}_{3}$ aerogel sample at a temperature of $4.2 \mathrm{~K}$ using a nuclear magnetic resonance field gradient technique. A strong influence of ${ }^{3} \mathrm{He}$ adsorption in the aerogel on self-diffusion is observed. The classical consideration of adsorptive gas diffusion in mesopores leads to anomalously high tortuosity factors. The application of a more sophisticated model than the simple combination of empirical two-phase diffusion and the Knudsen gas diffusion models is required to explain our results. Anisotropic properties of the aerogel are not reflected in the observed gas diffusion even at low gas densities where the anisotropic Knudsen regime of diffusion is expected. The observed gas densification indicates the influence of the aerogel attractive potential on the molecular dynamics, which probably explains the reduced diffusion process. Perhaps this behavior is common for any adsorptive gases in nanopores.
\end{abstract}

\section{Introduction}

Diffusion study is a widely used tool for the characterization of porous media. ${ }^{1}$ The understanding of fluid dynamics in nano and mesoporous materials is required for the correct interpretation of diffusion data. Particularly, gas adsorption/desorption processes play a significant role in gas dynamics.

Aerogels with open-space pores present unique model systems for diffusion studies due to a rich variety of available structures. Recently the interest to study superfluids in aerogels also arose due to the observation of polar superfluid phases in nematically ordered aerogels. ${ }^{2,3}$ The ballistic mean-free path (mfp) in an aerogel $\lambda_{\text {aero }}$ is an important parameter for theoretical models of ${ }^{3} \mathrm{He}$ superfluidity and can be determined by diffusion experiments, for instance, via magnetic resonance with pulse gradients. At very low temperatures $(<10 \mathrm{mK})$ the density of quasiparticles in liquid is so small that its $\mathrm{mfp}$ is defined by quasiparticle-aerogel collisions ${ }^{4-6}$ and coincides with the ballistic mean-free path in the aerogel. Under these conditions the self-diffusion of quasiparticles can be considered as Knudsen diffusion. Recently Dmitriev et al. ${ }^{7}$ have reported the observation of strongly anisotropic Knudsen diffusion of liquid ${ }^{3} \mathrm{He}$ at

\footnotetext{
${ }^{a}$ Kazan Federal University, Kazan, 420008, Russian Federation. E-mail:slava625@yandex.ru, kajum@inbox.ru; Fax: +7 843233 7355; Tel: +78432337306

${ }^{b}$ Institute of Perspective Research, Academy of Sciences of the Republic of Tatarstan, Kazan 420111, Russia
}

1-10 $\mathrm{mK}$ in a new type of aerogel (Nafen-90) with almost parallel strands.

Information on the mfp in aerogels may also be obtained from room temperature diffusion experiments with hyperpolarized gases, ${ }^{8}$ such as ${ }^{3} \mathrm{He}$ or ${ }^{129} \mathrm{Xe}$. Diffusion measurements with Boltzmann-polarized gases at room temperature are also feasible but require a high gas density or the application of a high magnetic field for a sufficient signal-to-noise ratio. The high density is available, for instance, for strongly adsorbing gases for which a significant fraction of the atoms are in an adsorbed layer with reduced mobility. However in that case the adsorption should be taken into account. ${ }^{9,10}$ Lee et $a l .{ }^{11}$ reported diffusion experiments of methanol gas significantly adsorbed in slightly anisotropic aerogel samples at room temperature with the aim to characterize the mfp for subsequent studies of superfluid ${ }^{3} \mathrm{He}$ in this aerogel. An empirical model based on "fast exchange", that accounts for the adsorbed layer was applied to determine the gas diffusion in the aerogel, which was assumed to be in the Knudsen diffusion regime. On the other hand, Mueller et $a l^{10}$ applied a similar model to $\mathrm{CO}_{2}$ diffusion in aerogels with additional tortuoisity factors for Knudsen and free gas diffusion. Although such experiments at room temperatures are more feasible in most NMR laboratories, some specific features can make them more problematic to interpret.

The effect of attractive forces from aerogel strands can lead to the formation of adsorbed layers and to a modification of the gas molecule trajectories and therefore to a diffusion suppression of low density gas in nanoscale diameter pores. ${ }^{12}$ Alternatively, liquid 
${ }^{3} \mathrm{He}$ (with surface pre-plating with ${ }^{4} \mathrm{He}$ ) at very low temperatures and room temperature hyperpolarized ${ }^{3} \mathrm{He}$ diffusion can be accurately described by the Knudsen model which gives direct information on the mfp. This is due to the absence of the wall attractive potential on quasiparticles in liquid ${ }^{3} \mathrm{He}$ or its small influence in ${ }^{3} \mathrm{He}$ gas at room temperature. Thus the diffusion of free gas in the presence of adsorption cannot be considered as simple Knudsen diffusion even with a low fraction for the adsorbed layer.

A more advanced model for the transport of gases at low density in simple pore geometries named the "oscillator model" was built by Bhatia et al., ${ }^{12}$ and considers the atom oscillation movements between walls in the fluid-solid attractive potential of the pore and shows excellent agreement with molecular dynamics simulations. It was shown by the authors of this study that the Knudsen model can significantly overpredict diffusion in the nanoscale diameter pore even at high temperatures. ${ }^{13,14}$ The "oscillator model" is applicable in the case of negligible particle-particle interaction, which is usual for low gas densities and Henry's adsorption regime, whereas diffusion of high density adsorbing gases still remains poorly studied.

In this article we report low temperature ${ }^{3} \mathrm{He}$ strongly adsorbed gas diffusion experiments in a broad range of gas pressures (and densities) in nematically ordered aerogel (Nafen-90) in which the new superfluid ${ }^{3} \mathrm{He}$ phase was observed. ${ }^{3,7}$ The aims of our study are to examine empirical diffusion models likewise applied by Lee et $a{ }^{11}{ }^{11}$ and Mueller et $a l^{10}$ at various conditions and to assess the ordered aerogel structure. This is the first report on low temperature ${ }^{3} \mathrm{He}$ gas diffusion measurements in nanostructures to our knowledge.

\section{Experimental}

\subsection{Aerogel sample and helium-3 management}

The nematically ordered Nafen aerogel with $90 \mathrm{mg} \mathrm{\textrm {cm } ^ { - 3 }}$ density produced by ANF technology was used as a sample. It consists of relatively long (a few centimeters) parallel $\gamma-\mathrm{Al}_{2} \mathrm{O}_{3}$ nano fibers of $8 \mathrm{~nm}$ diameter; detailed information on its properties was presented by Asadchikov et al. ${ }^{15}$ The porosity of this sample is estimated to be $97.8 \%$. The SEM image of the sample (Fig. 1) was obtained by Mikhail Presnyakov on a Titan 80-300 S/TEM (FEI, USA) at Probe and Electron microscopy RC "NANOPROBE", National Research Center "Kurchatov Institute", Moscow. The sample length in our experiments is $2.5 \mathrm{~cm}$. It was tightly inserted into a Pyrex glass cell (5 mm i.d.). The sample fibers were oriented along the experimental cell $(x)$ and perpendicular to the external magnetic field $(z)$ as shown in Fig. 2. The experimental cell was sealed to the ${ }^{3} \mathrm{He}$ capillary. The sample and the cell were flushed several times with helium gas and pumped out before experiments. The ${ }^{4} \mathrm{He}$ concentration in the ${ }^{3} \mathrm{He}$ used was less than $0.01 \%$. The gas pressure was measured in a dead volume at room temperature by a Pfeiffer Vacuum RPT-200 Piezo/Pirani pressure gauge with a specially measured calibration curve for ${ }^{3} \mathrm{He}$ gas. The estimated thermomolecular pressure

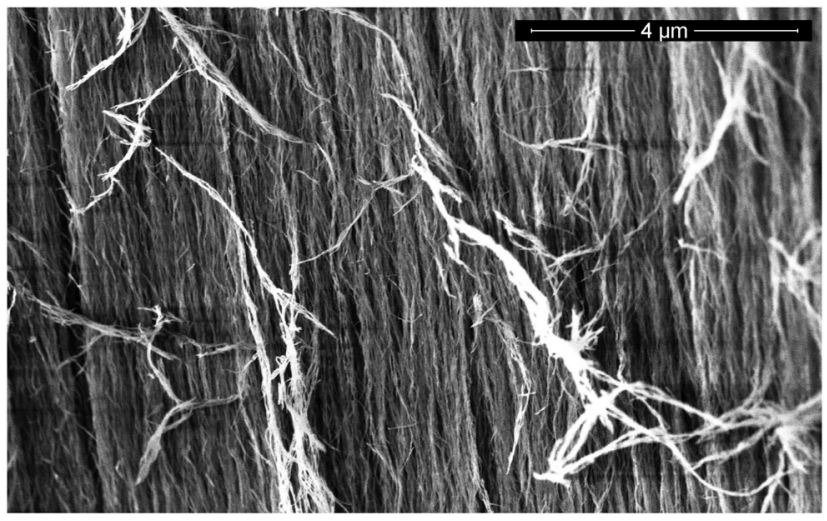

Fig. 1 The SEM image of the ordered Nafen aerogel.

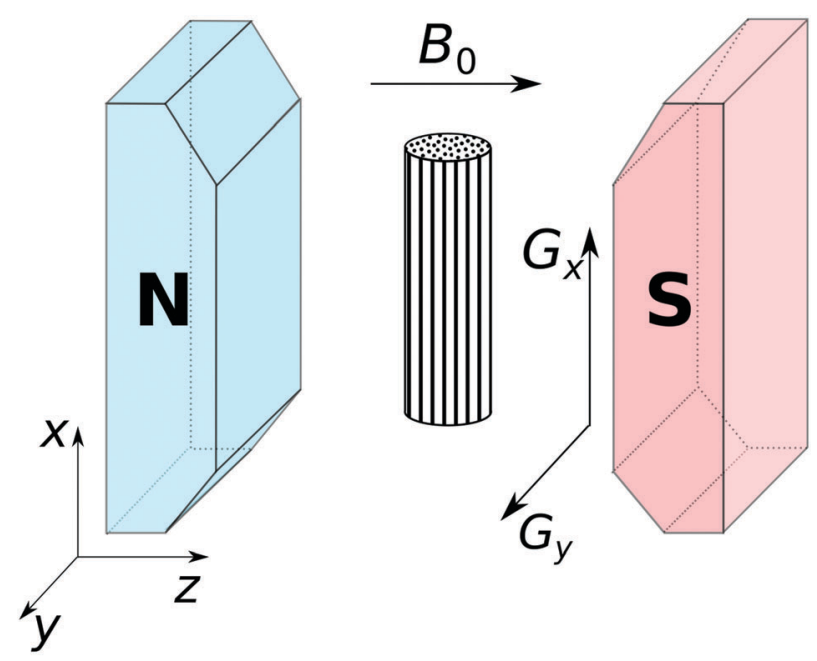

Fig. 2 The schematic illustration of the relative directions of magnetic field, applied gradients and aerogel fibers in our experiments.

difference between the room temperature dead volume and the low temperature cell volume in our experiments was negligible.

\subsection{NMR apparatus and gradient coil}

A home-built pulsed NMR spectrometer,${ }^{16}$ along with a resistive magnet (up to $0.8 \mathrm{~T}$ ) and a glass helium cryostat, was used in the NMR diffusion experiments. A BKPrecision XLN-8018 power supply was used to drive the resistive magnet. A single layer solenoidal NMR detection coil was wound on the cell surface with a $0.25 \mathrm{~mm}$ copper wire. The resonance circuit was tuned to a frequency of $16.4 \mathrm{MHz}$ and matched to $50 \Omega$ by ceramic capacitors located outside of the helium cryostat, and had a quality factor of $Q=91$. A low-noise fast response Miteq AU-1549 rf preamplifier was used for NMR detection. Typical signal-to-noise ratio values in our experiments were in the range of 15 to 140 .

Anti-Helmholtz gradient coils were wound with a $0.45 \mathrm{~mm}$ copper wire on the cylindrical polyester/epoxy shell $(5.2 \mathrm{~cm}$ i.d., $5.4 \mathrm{~cm}$ o.d.) and consisted of 87 turns for every 5 layers. The distance between the coils was set to $5.3 \mathrm{~cm}$ in order to fit 
outside of the glass cryostat used $(5.2 \mathrm{~cm}$ o.d.). The design of the coil shell allowed us to apply gradients in parallel ( $x$ axis) and perpendicular ( $y$ axis) directions to the fibers of the sample while the direction of the field $B_{0}$ was along the $z$ axis (Fig. 2). The pair of coils $\left(1.1 \mathrm{mT} \mathrm{cm}^{-1} \mathrm{~A}^{-1}\right)$ produced a constant $90 \%$-homogeneity gradient of magnetic field $\partial B_{0} / \partial x$ or $\partial B_{0} / \partial y$ over the sample length. The magnetic field gradients and inhomogeneity were deduced from additional $\mathrm{H}_{2} \mathrm{O}$ NMR experiments at room temperature. The gradient coils were placed on the outside of the glass-made cryostat and were driven by two Aktakom APS-7305 power supplies connected in series. The maximum available current for this configuration is $3 \mathrm{~A}$ which induces a magnetic field gradient stronger than the local $B_{0}$ residual field inhomogeneity by two orders of magnitude. The gradient current was triggered by a combination of Goodsky RW-SH-103D relays which were controlled via TTL pulse during a rf pulse sequence in the NMR experiments. The gradient was turned on for $1 \mathrm{~s}$ prior to any $\mathrm{rf}$ pulse to avoid the influence of eddy currents generated in the metal shield of the NMR probe on the detected signal.

\subsection{NMR diffusion measurements}

A standard Hahn echo sequence ( $4.8 \mu \mathrm{s}-\tau-9.6 \mu \mathrm{s})$ was used to measure the ${ }^{3} \mathrm{He}$ nuclei transverse magnetization relaxation time $T_{2}$ in the self-diffusion measurements. Spin echo amplitude decays were obtained at $2.2 \mathrm{mT} \mathrm{cm}^{-1}$ and $3.3 \mathrm{mT} \mathrm{cm}^{-1}$ magnetic field gradient values for two directions: parallel $(x)$ and perpendicular $(y)$ to the sample fibers, but always perpendicular to the external magnetic field $(z)$. The obtained spin echo decay curves were fitted by the following function: ${ }^{17}$

$$
S(2 \tau)=S_{0} \exp \left(-2 \tau / T_{2}\right) \exp \left(-A(2 \tau)^{3}\right),
$$

where $S(2 \tau)$ is the spin echo amplitude at time $t=2 \tau, S_{0}$ is the signal amplitude corresponding to total magnetization, and $T_{2}$ is the ${ }^{3} \mathrm{He}$ nuclei transverse magnetization relaxation time. The parameter $A$ for a given gradient $G$, diffusion coefficient $D$ and ${ }^{3} \mathrm{He}$ gyromagnetic ratio $\gamma=2 \pi \times 32.43 \mathrm{MHz} \mathrm{T}^{-1}$ can be written as:

$$
A=\gamma^{2} D G^{2} / 12 .
$$

The spin-lattice relaxation time $T_{1}$ of the ${ }^{3} \mathrm{He}$ nuclei was measured by a saturation-recovery method and exceeded the observed $T_{2}$ times by two orders of magnitude. ${ }^{18}$

\section{Results}

Fig. 3 (circle symbols) shows the ${ }^{3} \mathrm{He}$ amount in the cell $N_{0}$ as computed using the measured NMR signal amplitudes versus pressure $P$ in the pure ${ }^{3} \mathrm{He}$ experiments. The signal amplitudes were measured using the spin echo technique with corresponding delays between the $\pi$ and $\pi / 2$ pulses of $\tau=100 \mu \mathrm{s}$, much shorter than $T_{2}$ (few milliseconds). It is known that ${ }^{3} \mathrm{He}$ forms a monolayer film on the substrate surface at a temperature of $4.2 \mathrm{~K}$, and that the adsorbed ${ }^{3} \mathrm{He}$ amount is almost independent of pressure above 50 mbar and varies within $20 \%$ above 1 mbar. ${ }^{19-21}$ Therefore it was



Fig. 3 Amount of ${ }^{3} \mathrm{He}$ in the experimental cell containing $\mathrm{Al}_{2} \mathrm{O}_{3}$ aerogel obtained by NMR measurements of Hahn echo amplitude versus ${ }^{3} \mathrm{He}$ gas pressure $P$ at $4.2 \mathrm{~K}$. Solid lines represent extrapolation of the high pressure region linear fits and their intercepts provide the amount of adsorbed ${ }^{3} \mathrm{He}$ : $6.4 \pm 0.5 \mathrm{scc}$ in pure ${ }^{3} \mathrm{He}$ and $1.3 \pm 0.3 \mathrm{scc}$ in ${ }^{3} \mathrm{He}-{ }^{4} \mathrm{He}$ mixture experiments.

assumed that at high enough pressures the amount of adsorbed ${ }^{3} \mathrm{He}$ is almost constant (after completion of the monolayer) and so the signal amplitude is linear to the amount of ${ }^{3} \mathrm{He}$ in the gas phase. The linear fit gives the slope $k$ which corresponds to the dependence $N_{\text {gas }}(P)=k P$ and capacity of the adsorbed layer $\left(N_{\text {ads }}\right)$ which corresponds to the extrapolation of a linear fit to zero pressure. Using that, one can estimate the ratio of $N_{\text {gas }}$ to the total ${ }^{3} \mathrm{He}$ amount $N_{0}$ at any pressure:

$$
\frac{N_{\text {gas }}}{N_{0}}=\frac{k P}{k P+N_{\text {ads }}} .
$$

The absolute density of the gas in the aerogel was found from the volume occupied by gas in the aerogel (taking into account the porosity of the aerogel) and by the calibration of the NMR signal from the adsorbed layer $(P=1.6 \mathrm{mbar}$ at which the gaseous phase is negligible), this corresponds to $6.16 \mathrm{scc}$ of gas condensed from the calibrated volume at room temperature. The obtained monolayer capacity $N_{\text {ads }}$ (see Fig. 3 ) corresponds to $\approx 16.35 \mathrm{~m}^{2}$ surface area assuming a ${ }^{3} \mathrm{He}$ monolayer density ca. $9.4 \times 10^{18}$ atoms per $\mathrm{m}^{2}$ (ref. 22) which agrees well with that determined for the same sample by Brunauer-Emmett-Teller analysis of a $\mathrm{N}_{2}$ isotherm at $77 \mathrm{~K}\left(16.1 \mathrm{~m}^{2}\right)$. We found that the density of the gas phase in the aerogel is $2.03 \pm 0.03$ times higher than that of free ${ }^{3} \mathrm{He}$ gas at $4.2 \mathrm{~K}$. The typical gaseous ${ }^{3} \mathrm{He}$ transverse magnetization decay curve in our experiments without application of a magnetic field gradient is shown in Fig. 4 (open symbols). The measured spin echo decay curves are slightly affected by spin diffusion in the magnetic field inhomogeneity of the resistive magnet but still allow us to obtain reliable $T_{2}$ values. The measured $T_{2}$ values as a function of gas pressure are displayed in Fig. 5 . The ${ }^{3} \mathrm{He}$ transverse relaxation time approximately increases linearly with the gas pressure. This dependence points out a strong influence of the 


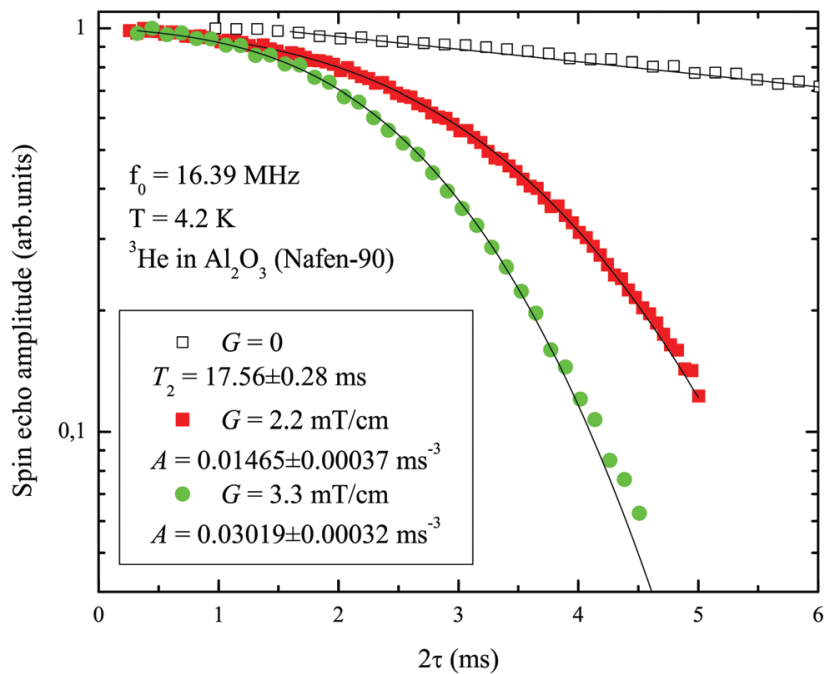

Fig. 4 Typical ${ }^{3} \mathrm{He}$ spin echo amplitude decay curves with $\left(G=2.2 \mathrm{mT} \mathrm{cm}^{-1}\right.$ and $3.3 \mathrm{mT} \mathrm{cm}^{-1}$ ) and without an applied magnetic field gradient obtained in pure ${ }^{3} \mathrm{He}$ experiments at $770 \mathrm{mbar}$ gas pressure and at $4.2 \mathrm{~K}$. The measured ${ }^{3} \mathrm{He}$ transverse magnetization relaxation time $T_{2}=17.56 \pm 0.28 \mathrm{~ms}$ is much longer than the probed range of $2 \tau$. Solid lines represent data fits using eqn (1).

adsorbed layer on the ${ }^{3} \mathrm{He}$ gas transverse magnetization relaxation as was already found for silica aerogel. ${ }^{23}$

The obtained $T_{2}$ values were used to fit the spin echo amplitude decay curves measured with an applied gradient using eqn (1), with the variable $A$ parameter. Typical measured echo decay curves are presented in Fig. 4 for two gradient values. Application of 2.2 and $3.3 \mathrm{mT} \mathrm{cm}{ }^{-1}$ gradients significantly decreases the echo amplitude decay time. The diffusion coefficient $D$ was then estimated using eqn (2).

The measured ${ }^{3} \mathrm{He}$ diffusion coefficient $D$ dependencies on the ${ }^{3} \mathrm{He}$ gas pressure $P$ at $4.2 \mathrm{~K}$ temperature for two different gradient values are shown in Fig. 6 . The observed short $T_{2}$ values allowed us to perform diffusion experiments. The obtained ${ }^{3} \mathrm{He}$

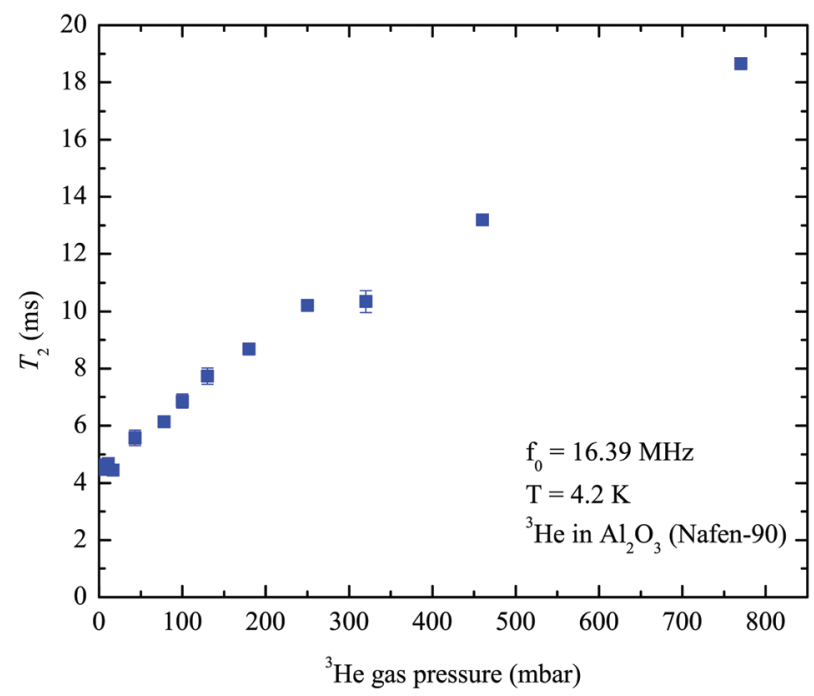

Fig. $5{ }^{3} \mathrm{He}$ transverse relaxation times $T_{2}$ measured at various ${ }^{3} \mathrm{He}$ gas pressures at $4.2 \mathrm{~K}$ in the pure ${ }^{3} \mathrm{He}$ experiment.

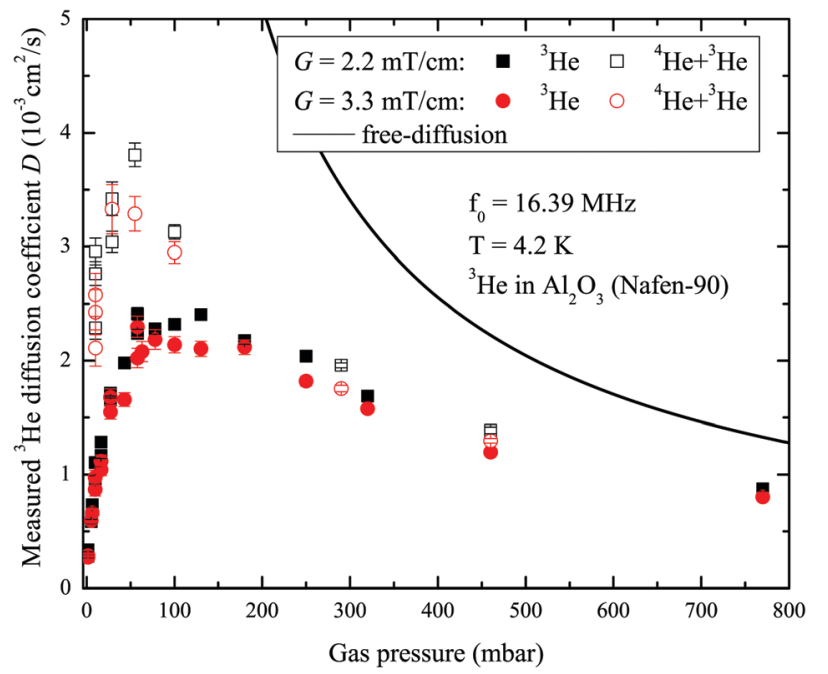

Fig. 6 Pressure dependence of measured ${ }^{3} \mathrm{He}$ diffusion coefficients $D$ obtained in pure ${ }^{3} \mathrm{He}\left(G=2.2 \mathrm{mT} \mathrm{cm}{ }^{-1}\right.$ and $\left.3.3 \mathrm{mT} \mathrm{cm}^{-1}\right)$ and in ${ }^{3} \mathrm{He}-{ }^{4} \mathrm{He}$ mixture $\left(G=2.2 \mathrm{mT} \mathrm{cm}^{-1}\right.$ and $\left.3.3 \mathrm{mT} \mathrm{cm}^{-1}\right)$ in $\mathrm{Al}_{2} \mathrm{O}_{3}$ aerogel at $4.2 \mathrm{~K}$. The solid line is the expected diffusion (eqn (4)) for ${ }^{3} \mathrm{He}$ gas with a density determined from the linear fit in Fig. 3.

diffusion coefficient $D$ values do not vary with the applied gradient. This is additional evidence that gradient coils create higher magnetic field gradients than that of the resistive magnet in our experiments, therefore the influence of residual gradients on the measured $D$ values is negligible and the obtained values are correct. The measured diffusion coefficients $D$ differ by two orders of magnitude from the one of a free gas at low pressures (see Fig. 6). For the given diffusion coefficients and the measurement durations used the probed range of diffusion lengths $(\sqrt{2 D \tau})$ in our experiments varies from $14 \mu \mathrm{m}$ to $28 \mu \mathrm{m}$.

The measured pressure dependence of ${ }^{3} \mathrm{He}$ diffusion is not typical. The anomalous drop in the diffusion coefficient at low pressures is due to the influence of the adsorptive effect of the aerogel whereas at high pressures the measured diffusion coefficient asymptotically converges to the gas diffusion computed for the estimated density. The expected free gas diffusion in the aerogel at a given density was found from the experimentally determined dependence $D(\rho)$ at $4.2 \mathrm{~K}$ by Luszczynski ${ }^{24}$ for a free gas:

$$
D \rho=17.5 \times 10^{-6} \mathrm{~g} \mathrm{~s}^{-1} \mathrm{~cm}^{-1} .
$$

Note that computed in this way, diffusion in densified gas in an aerogel does not coincide with free diffusion at any given pressure, because we assume that diffusion is a function of the gas density and not of the pressure.

Additional experiments with a ${ }^{4} \mathrm{He}$ aerogel coating were performed in order to emphasise the role of the adsorbed ${ }^{3} \mathrm{He}$ layer in apparent diffusion at low pressures (see Fig. 3 and 6). We also observe the increase of the ${ }^{3} \mathrm{He}$ density in the gas phase in the aerogel (by a factor of $1.87 \pm 0.03$ higher than that of free ${ }^{3} \mathrm{He}$ gas at $4.2 \mathrm{~K}$ ), but the adsorbed amount of ${ }^{3} \mathrm{He}$ is much smaller compared to that of the pure ${ }^{3} \mathrm{He}$ data (Fig. 3). Precoating the aerogel surface with one layer of ${ }^{4} \mathrm{He}$ significantly 
increases the diffusion of ${ }^{3} \mathrm{He}$ at low pressures, but at high pressures ${ }^{3} \mathrm{He}$ diffusion converges with the diffusion observed in the pure ${ }^{3} \mathrm{He}$ experiment (Fig. 6).

Experiments to probe the diffusion anisotropy were performed for pure ${ }^{3} \mathrm{He}$ and the ${ }^{3} \mathrm{He}-{ }^{4} \mathrm{He}$ mixture as well. The ratio of the ${ }^{3} \mathrm{He}$ diffusion coefficients $D$ measured at gradients parallel $\left(D^{\|}\right)$ and perpendicular $\left(D^{\perp}\right)$ to the aerogel fibers is presented in Fig. 7 . Diffusion anisotropy is not observed in these experiments even at low pressures at which the Knudsen regime of diffusion is expected.

\section{Discussion}

The measured apparent diffusion coefficient of ${ }^{3} \mathrm{He}$ in the aerogel is significantly suppressed by the immobile adsorbed layer of ${ }^{3} \mathrm{He}$ (the estimated diffusion coefficient in the adsorbed layer lies between $10^{-9}$ and $10^{-4} \mathrm{~cm}^{2} \mathrm{~s}^{-1}$ depending on the layer coverage $\left.\mathrm{e}^{25,26}\right)$. This is demonstrated in Fig. 6, where the diffusion coefficient anomalously drops at low pressures in opposition to that expected for free gas diffusion. It is also confirmed in experiments with the ${ }^{3} \mathrm{He}-{ }^{4} \mathrm{He}$ mixture: the covering of the aerogel surface with an approximate monolayer of ${ }^{4} \mathrm{He}$ significantly increases the gaseous ${ }^{3} \mathrm{He}$ diffusion coefficient at low pressures as can be seen from Fig. 6. This occurs due to a preferential adsorption of ${ }^{4} \mathrm{He}$ on the aerogel surface and therefore a high fraction of gas phase ${ }^{3} \mathrm{He}$ in the total ${ }^{3} \mathrm{He}$ amount. Nevertheless the ${ }^{3} \mathrm{He}$ diffusion is much slower than that expected for the bulk ${ }^{3} \mathrm{He}$ free gas which can partly be explained by the presence of a small ${ }^{3} \mathrm{He}$ fraction in the adsorbed layer on the aerogel surface. At high pressures the effect of the ${ }^{4} \mathrm{He}$ adsorbed layer on the ${ }^{3} \mathrm{He}$ diffusion is not significant and therefore $D(P)$ coincides with that of pure ${ }^{3} \mathrm{He}$ in the aerogel. Similarly to our result, Lee et al. ${ }^{11}$ reported the observation of the "anomalous" diffusion of methanol in aerogels due to the effect of adsorbed layers in the aerogels at

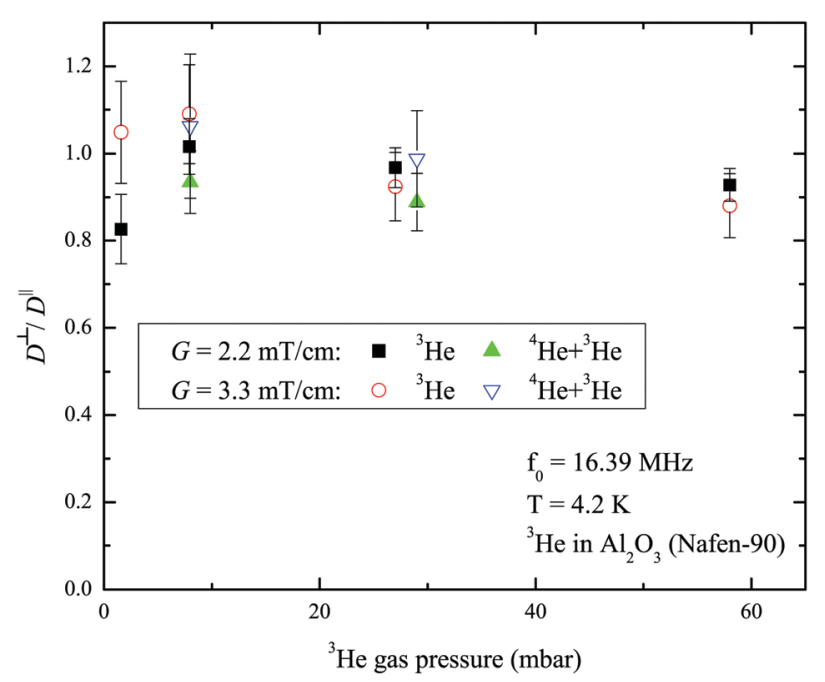

Fig. 7 Relation between measured ${ }^{3} \mathrm{He}$ diffusion coefficient values obtained at magnetic field gradients perpendicular $\left(D^{\perp}\right)$ and parallel $\left(D^{\|}\right)$ to aerogel fibers. Diffusion coefficient $D$ values are measured for ${ }^{3} \mathrm{He}$ and the ${ }^{3} \mathrm{He}-{ }^{4} \mathrm{He}$ mixture at $4.2 \mathrm{~K}$. room temperatures. Following ref. 9 and 11, the apparent diffusion in this aerogel is just weighted diffusion between that in the adsorbed layer and that in the gas phase. As diffusion in the gas phase is much faster than in the adsorbed layer the apparent diffusion in the aerogel is assumed to be governed by a "fast exchange" process: ${ }^{4}$

$$
D=D_{\text {gas }}^{\text {aero }} \frac{N_{\text {gas }}}{N_{0}}+D_{\text {surf }} \frac{N_{\text {ads }}}{N_{0}},
$$

where $D_{\text {gas }}^{\text {aero }}$ is the diffusion coefficient of the ${ }^{3} \mathrm{He}$ gas phase inside the aerogel, and $D_{\text {surf }}$ is the diffusion coefficient of the adsorbed ${ }^{3} \mathrm{He}$. The approach of the two-phase fast-exchange model is valid if the lifetimes in each phase are much shorter than the measurement timescale. ${ }^{27}$ This condition is satisfied in our experiments as the estimated typical ${ }^{3} \mathrm{He}$ atom lifetime in the adsorbed layer is $\sim 1 \mathrm{~ns}$, and is $\sim 10 \mathrm{~ns}$ in the gas phase; $\dagger$ both values are much shorter than typical measurement times.

Note that the surface diffusion term which plays an important role $^{1,30}$ for some gases at high temperatures will be ignored further as being totally negligible even at very low gas fractions compared to the gas diffusion term in our experiment. This is based on the fact that the diffusion coefficient in the ${ }^{3} \mathrm{He}$ surface film is of orders $10^{-9}-10^{-8} \mathrm{~cm}^{2} \mathrm{~s}^{-1}$ for high monolayer coverages at the experimental temperature. ${ }^{26,31,32}$ According to eqn (5), one can estimate diffusion in the gas phase inside the aerogel $\left(D_{\text {gas }}^{\text {aero }}\right)$, taking into account its fraction. This was done for the apparent diffusion data presented in Fig. 6 using adsorption data from Fig. 3 and applying eqn (3). A similar procedure was applied to the results of the ${ }^{3} \mathrm{He}-{ }^{4} \mathrm{He}$ experiments. The obtained results are shown in Fig. 8 where the expected diffusion for free gas is also plotted with density determined from the data of the measured isotherms (Fig. 3) and a known cell volume. It can be seen that in the whole range of $P$, the computed diffusion is significantly lower than expected especially at low pressures at which the fraction of adsorbed atoms is large. The computed diffusion $D_{\text {gas }}^{\text {aero }}$ values for the pure ${ }^{3} \mathrm{He}$ experiments and ${ }^{3} \mathrm{He}-{ }^{4} \mathrm{He}$ mixtures are close to each other. This validates the correctness of applying the fast-exchange model (eqn (5)) in computing $D_{\text {gas }}^{\text {aero }}$. This is also clear and strong evidence that the observed effect is almost independent of the fraction $N_{\text {ads }}$ of adsorbed ${ }^{3} \mathrm{He}$ as ${ }^{4} \mathrm{He}$ is known to preferentially occupy the sample surface compared to ${ }^{3} \mathrm{He}$. The presence of ${ }^{4} \mathrm{He}$ significantly decreases the amount of adsorbed ${ }^{3} \mathrm{He}$ by a factor of $c a$. 5 . Therefore the observed effect of slow diffusion $D_{\text {gas }}^{\text {aero }}$ cannot be explained by the influence of surface diffusion.

On the other hand, the estimated gas density in the aerogel using eqn (3) and data from Fig. 3 is somewhat $2.03 \pm 0.03$ times higher than computed for the ideal gas at any given pressure. Reliability of the estimated density by NMR calibration is confirmed by the expected diffusion (for a given density) approaching

$\dagger$ The lifetime of a ${ }^{3} \mathrm{He}$ atom in the adsorbed layer is determined by eqn (12) of Lusher $e$ t al. ${ }^{28}$ at $4.2 \mathrm{~K}$, a measured aerogel surface of $16.1 \mathrm{~m}^{2}$, a void volume based on an empty cell volume of $0.49 \mathrm{~cm}^{3}$ and aerogel porosity, and a sticking probability of about $1 .{ }^{29}$ The lifetime of a ${ }^{3} \mathrm{He}$ atom in the gas phase is roughly determined by successive collisions of ${ }^{3} \mathrm{He}$ atoms with aerogel fibers. It is on the scale of $\lambda_{\text {aero }}{ }^{2} / D_{\text {gas }}^{\text {aero }}$, where $\lambda_{\text {aero }}$ is determined by Dmitriev et al. ${ }^{3}$ 


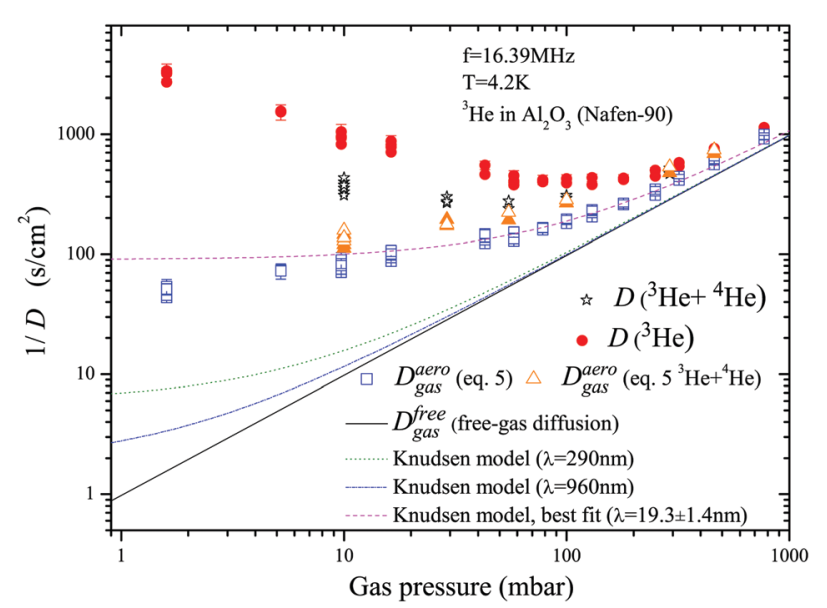

Fig. 8 Inverse diffusion gas pressure dependencies and computed $D_{\text {gas }}^{\text {aero }}$ using data from Fig. 3 and applying eqn (5). The dashed lines show expected free gas diffusion (gas densification is accounted) and the Knudsen gas diffusion in the aerogel with $\lambda_{\text {aero }}=290 \mathrm{~nm}$ and $960 \mathrm{~nm}$, which correspond to two principal values of lambda obtained for this aerogel $^{7}$ and $\lambda_{\text {aero }}=19.3 \pm 1.4 \mathrm{~nm}$ which fits the experimental data.

the experimental diffusion at high pressures (Fig. 6). This occurs when the adsorbed layer fraction becomes negligible, whereas the estimated diffusion of the ideal bulk gas at high pressures is about 3 times higher than measured (not presented). Mueller et al. ${ }^{10}$ also observed adsorbate densification of $\mathrm{CO}_{2}$ by a factor of $\approx 2$ in an aerogel. They refer this effect to the adsorption of $\mathrm{CO}_{2}$ in micropores. In the case of helium at low temperatures it is known that adsorption in micropores is completed at relatively low pressures of a few mbars. ${ }^{33,34}$ At higher pressures the excess amount of helium contributes only to the gas phase. Thus the possible source of the observed densification does not involve an additional increase in the adsorbed layer density in our case. The reason for such gas compression could be the modification of gas properties inside the aerogel because the aerogel wall adsorption potential spreads far from the first and the second ${ }^{3} \mathrm{He}$ adsorbed layers on the aerogel surface. Because of that, one can expect a nonuniform gas density depending on the distance from the aerogel strands. Such an effect of gas densification was also found, for instance, in simulations of nitrogen filling nanotubes with diameters of $0.8-6.3 \mathrm{~nm}$ at room temperature. $^{35}$

Another source of reduced apparent diffusion in the aerogel is the ${ }^{3} \mathrm{He}$ atom collisions with the aerogel strands. This is known as the Knudsen diffusion which appears when the mfp in the gas phase, due to atom-atom collisions, is longer or of the order of the geometrical ballistic mfp in an aerogel $\left(\lambda_{\text {aero }}\right)$. In that case the diffusion coefficient in the gas phase in an aerogel is usually computed as: ${ }^{9-11}$

$$
\frac{1}{D_{\mathrm{gas}}^{\mathrm{aero}}}=\frac{1}{D_{\mathrm{gas}}^{\mathrm{free}}}+\frac{1}{D_{\mathrm{Kn}}},
$$

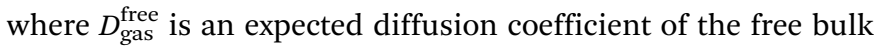
gas at a given helium density,

$$
D_{\mathrm{Kn}}=\frac{1}{3} \lambda_{\text {aero }} V_{\text {gas }},
$$

is the Knudsen diffusion coefficient. $V_{\text {gas }}$ is the mean ${ }^{3} \mathrm{He}$ atom velocity in an ideal gas:

$$
V_{\text {gas }}=\sqrt{\frac{8 k_{\mathrm{B}} T}{\pi m}},
$$

where $m$ is the ${ }^{3} \mathrm{He}$ atomic mass and $k_{\mathrm{B}}$ is the Boltzmann constant.

Our diffusion measurements were performed in the gaseous phase far from the Knudsen regime (except for very low pressures) because of sufficiently low temperatures and high gas densities, for which the mfp in the gas phase is much shorter than the expected characteristic length in this aerogel.

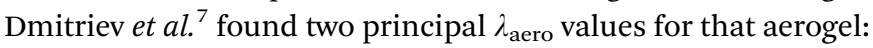
$\lambda_{\text {aero }}^{\perp}=290 \mathrm{~nm}$ and $\lambda_{\text {aero }}^{\|}=980 \mathrm{~nm}$. Using these parameters we have plotted the expected $1 / D_{\text {gas }}^{\text {aero }}$ values in Fig. 8 . As it can be seen these plotted curves lie significantly lower than the experimental ones. It was suggested by Tastevin and Nacher ${ }^{8}$ that in order to explain diffusion data in an aerogel it is necessary to introduce the broad spectrum of $\lambda_{\text {aero }}$ which lies within the range of 5-1000 $\mathrm{nm}$. To explain our diffusionpressure dependence using the Knudsen regime we must introduce very short $\lambda_{\text {aero }}$ of the order of $20 \mathrm{~nm}$ which seems to be unreasonable taking into account the high porosity of our aerogel (Fig. 8).

Mueller et al. ${ }^{10}$ suggested using a modified model based on eqn (6) with introduced tortuosity factors for Knudsen diffusion $\eta_{\mathrm{K}}$ and for free gas in an aerogel $\eta_{\mathrm{g}}$ :

$$
\frac{1}{D_{\mathrm{gas}}^{\text {aero }}}=\frac{\eta_{\mathrm{g}}}{D_{\mathrm{gas}}^{\text {free }}}+\frac{\eta_{\mathrm{K}}}{D_{\mathrm{Kn}}} .
$$

In general, the tortuosity factors are meant to account for the complex structure of the porous media. The application of this model to our data using the above mentioned $\lambda_{\text {aero }}^{\|}$and $\lambda \stackrel{\perp}{\perp}$ does provide enormously high tortuosity factors $\eta_{\mathrm{g}}=1.24$ (gas densification is accounted for) and $\eta_{\mathrm{K}}$ of 11.7 and 38.7, respectively. Obviously very high obtained tortuosity values $\eta_{\mathrm{K}}$ and a large difference between $\eta_{\mathrm{K}}$ and $\eta_{\mathrm{g}}$ point out the invalidity of such an approach. Mueller et al. ${ }^{10}$ also found high tortuosity values $\eta_{\mathrm{K}} \approx 6$ but they explain this by accounting for the adsorption in micropores which yields the observed densification of the adsorbate gas $\left(\mathrm{CO}_{2}\right)$ in the aerogel. As it was mentioned already our results do not involve additional adsorption and therefore this consideration cannot be applied.

In addition, diffusion measurements with parallel and perpendicular gradient orientations to the aerogel fibers show no difference (Fig. 7) even at very low pressures where diffusion is expected to be strongly anisotropic in the Knudsen regime due to anisotropy of the aerogel. Thus we must conclude that the correctness of the simple models application used by Lee et al. ${ }^{11}$ and Mueller et al. ${ }^{10}$ is doubtful. The observed diffusion of ${ }^{3} \mathrm{He}$ gas in the aerogel is significantly more suppressed by adsorption than expected. In addition, the Knudsen diffusion seems to be an inppropriate model to describe the diffusion of adsorptive gases. Similar problems of the straightforward application of the Knudsen model are discussed by Bhatia and Nicholson. ${ }^{14}$ 
Note that we were able to carefully test the $\operatorname{model}^{9,11}$ for a very broad range of $N_{\text {gas }} / N_{0}$ ratios (0.01-0.88) focusing on the adsorbed layer effect both far from the Knudsen regime at high pressures and at the Knudsen regime at low pressures, whereas Lee et al. are in the limit of a low gas fraction. The experimental conditions used are also different from those used in the studies of P.-J. Nacher and G. Tastevin ${ }^{8}$ who measured hyperpolarized gas diffusion in various silica isotropic aerogels for a broad range of pressures and at room temperature with a negligible adsorbed layer effect due to high temperature. Coming back to Lee's article we should say that the described analysis technique ${ }^{11}$ is very critical to the determination of the gas and the adsorbed layer densities. The described $\lambda$ anisotropy is highly probable to appear due to the methanol density uncertainty in experiments and is not connected with the properties of the aerogel geometry. The careful absolute gas density determination must be applied in order to exclude an additional free fit parameter of their fitting function. For instance, it seems to be unreasonable that their difference $\left(D^{\|}-D^{\perp}\right)$ changes sign depending on the pressure if one assumes single $\lambda^{\perp}$ and $\lambda^{\|}$parameters. Also $D^{\|}$and $D^{\perp}$ are expected to converge at high pressures which is not the case in their fits.

Thus, an appropriate model which takes into account the adsorbing effect of an aerogel instead of a simple atom-wall collision model (which leads to the Knudsen diffusion) should be developed. The developed "oscillator model",12 designed to describe the diffusion of adsorptive gases in simple pore geometries seems to be a good starting point to build such a model. Although it is not applicable in the case of high gas and adsorbed layer densities, it nevertheless shows that in the case of helium gas at low temperatures $(<30 \mathrm{~K})$ the error of the Knudsen model can exceed $300 \%$ for cylindrical pores with diameters of less than $20 \mathrm{~nm} .{ }^{13}$ It shows that the adsorption potential effect can be very strong at a temperature of $4.2 \mathrm{~K}$ even for $100 \mathrm{~nm}$ pores and its effect can not be accounted for by an empirical diffusion model of two exchanging phases (adsorbed layer-gas). Perhaps the described above complex adsorption effects in diffusion explain why we do not observe the diffusion anisotropy as Dmitriev et al. ${ }^{7}$ found at $20 \mathrm{mK}$ in liquid ${ }^{3} \mathrm{He}$ in this aerogel and their $\lambda_{\text {aero }}$ values do not describe our data within a combined Knudsen and two-phase fast-exchange model. This occurs due to the fundamental difference between the diffusion processes in adsorptive gases and low temperature quantum liquids confined in pores.

Besides, for a high signal to noise ratio at low temperatures even at a low ${ }^{3} \mathrm{He}$ gas density (unavailable for other gases at this temperature), the usage of ${ }^{3} \mathrm{He}$ to probe porous sample geometries and restricted diffusion provides advantages over other probe gases and allows one to increase the amount of obtained information on samples. Adsorbed layers play a significant role in ${ }^{3} \mathrm{He}$ gas diffusion and its influence can be varied or limited by ${ }^{4} \mathrm{He}$ surface coverage. The effect of adsorbed layers on diffusion can also be removed by $\mathrm{H}_{2}$ and following ${ }^{4} \mathrm{He}$ surface covering. ${ }^{28,36}$ Moreover, variation of the temperature allows one to change the ${ }^{3} \mathrm{He} \mathrm{mfp}$ values in a large range. Therefore aerogels and other porous samples can be studied under a wide range of experimental conditions that are unavailable using gases other than ${ }^{3} \mathrm{He}$. A high signal to noise ratio for ${ }^{3} \mathrm{He}$ gas NMR also proves ${ }^{3} \mathrm{He}$ as a convincing probe in diffusion experiments. Other gases used in aerogels such as methanol provide poor signal to noise ratios and one has to use adsorbed layers in order to increase the signal magnitude. ${ }^{11}$

\section{Conclusions}

We have performed ${ }^{3} \mathrm{He}$ spin echo gas diffusion measurements in a high porosity ordered aerogel sample at a temperature of 4.2 K. This is the first ${ }^{3} \mathrm{He}$ NMR measurement of gas diffusion in a restricted geometry at low temperatures. The strong influence of adsorbed ${ }^{3} \mathrm{He}$ layers on the aerogel is observed. An empirical model for accounting for adsorption by only considering the adsorbed layer ${ }^{9,11}$ does not correctly describe our data in the full range of pressures, and we have observed much slower diffusion than expected. Moreover, in contrast to the anisotropic diffusion of liquid ${ }^{3} \mathrm{He}$ recently found in this type of aerogel at very low temperatures ${ }^{7}$ at which quasiparticlequasiparticle or quasiparticle-aerogel collisions play a dominant role we do not observe anisotropic diffusion at low pressures in our highly ordered aerogel at $4.2 \mathrm{~K}$. This shows the significant effect of the adsorption attractive potential on atom dynamics in the gas phase and that application of the Knudsen diffusion model is incorrect in the case of strongly adsorbing gases at low temperatures. We found additional evidence of the strong influence of attractive potential in ${ }^{3} \mathrm{He}$ gas densification.

\section{Conflicts of interest}

There are no conflicts of interest to declare.

\section{Acknowledgements}

Authors are grateful to V. V. Dmitriev for the provided aerogel sample and inspiration for this work. Our thanks to A. V. Klochkov for the interest in this work and helpful discussions. This work was financially supported by the Russian Science Foundation (grant RSF 16-12-10359). The experimental part of this work was partially done on the equipment of the RC of Probe and EM (Kurchatov Complex of NBICS-Technologies, NRC “Kurchatov Institute”).

\section{References}

1 J. Kärger and R. Valiullin, Chem. Soc. Rev., 2013, 42, 4172. 2 N. Zhelev, M. Reichl, T. S. Abhilash, E. N. Smith, K. Nguyen, E. Mueller and J. M. Parpia, Nat. Commun., 2016, 7, 12975. 3 V. V. Dmitriev, A. A. Senin, A. A. Soldatov and A. N. Yudin, Phys. Rev. Lett., 2015, 115, 165304.

4 E. Collin, S. Triqueneaux, Y. M. Bunkov and H. Godfrin, Phys. Rev. B: Condens. Matter Mater. Phys., 2009, 80, 094422.

5 D. Candela and N. Kalechofsky, J. Low Temp. Phys., 1998, 113, 351. 
6 J. A. Sauls, Y. M. Bunkov, E. Collin, H. Godfrin and P. Sharma, Phys. Rev. B: Condens. Matter Mater. Phys., 2005, 72, 024507.

7 V. V. Dmitriev, L. A. Melnikovsky, A. A. Senin, A. A. Soldatov and A. N. Yudin, JETP Lett., 2015, 101, 808.

8 G. Tastevin and P.-J. Nacher, J. Chem. Phys., 2005, 123, 064506.

9 R. Valiullin, P. Kortunov, J. Kärger and V. Timoshenko, J. Phys. Chem. B, 2005, 109, 5746.

10 R. Mueller, S. Zhang, M. Klink, M. Baumer and S. Vasenkov, Phys. Chem. Chem. Phys., 2015, 17, 27481.

11 J. A. Lee, A. M. Mounce, S. Oh, A. M. Zimmerman and W. P. Halperin, Phys. Rev. B: Condens. Matter Mater. Phys., 2014, 90, 174501.

12 S. K. Bhatia, O. Jepps and D. Nicholson, J. Chem. Phys., 2004, 120, 4472.

13 M. R. Bonilla and S. K. Bhatia, J. Membr. Sci., 2011, 382, 339. 14 S. K. Bhatia and D. Nicholson, Chem. Eng. Sci., 2011, 66, 284.

15 V. E. Asadchikov, R. S. Askhadullin, V. V. Volkov, V. V. Dmitriev, N. K. Kitaeva, P. N. Martynov, A. A. Osipov, A. A. Senin, A. A. Soldatov, D. I. Chekrygina and A. N. Yudin, JETP Lett., 2015, 101, 556.

16 E. Alakshin, R. Gazizulin, A. Klochkov, V. Kuzmin, A. Sabitova, T. Safin and M. Tagirov, Magn. Reson. Solids, 2013, 15, 13104.

17 A. Abragam, The Principles of Nuclear Magnetism, Oxford University Press, Oxford, 1961.

18 E. M. Alakshin, M. Y. Zakharov, A. V. Klochkov, V. V. Kuzmin, K. R. Safiullin, A. A. Stanislavovas and M. S. Tagirov, JETP Lett., 2016, 104, 315.

19 J. G. Daunt and C. Z. Rosen, J. Low Temp. Phys., 1970, 3, 89.

20 M. Bretz, J. G. Dash, D. C. Hickernell, E. O. McLean and O. E. Vilches, Phys. Rev. A: At., Mol., Opt. Phys., 1973, 8, 1589.
21 C. P. Chen, S. Mehta, E. A. Hoefling, S. Zelakiewicz and F. M. Gasparini, J. Low Temp. Phys., 1996, 102, 31.

22 J. G. Daunt and E. Lerner, Monolayer and Submonolayer helium films, Springer, 1973.

23 A. V. Klochkov, V. V. Kuz'min, K. R. Safiullin, M. S. Tagirov, D. A. Tayurskii and N. Mulders, JETP Lett., 2008, 88, 823.

24 K. Luszczynski, R. E. Norberg and J. E. Opfer, Phys. Rev., 1962, 128, 186.

25 B. P. Cowan, M. G. Richards, A. L. Thomson and W. J. Mullin, Phys. Rev. Lett., 1977, 38, 165.

26 B. Cowan, M. Fardis, T. Crane and L. Abou-El-Nasr, Phys. B, 1990, 165-166, 707.

27 R. Kimmich, Principles of Soft-Matter Dynamics: Basic Theories, Non-invasive Methods, Mesoscopic Aspects, Springer, Netherlands, 2012.

28 C. P. Lusher, M. F. Secca and M. G. Richards, J. Low Temp. Phys., 1988, 72, 25.

29 M. Sinvani, M. W. Cole and D. L. Goodstein, Phys. Rev. Lett., 1983, 51, 188.

30 M. Dvoyashkin, A. Khokhlov, S. Naumov and R. Valiullin, Microporous Mesoporous Mater., 2009, 125, 58.

31 N. S. Sullivan, J. Low Temp. Phys., 1976, 22, 313.

32 D. R. Swanson, D. Candela and D. O. Edwards, J. Low Temp. Phys., 1988, 72, 213.

33 N. Setoyama and K. Kaneko, Adsorption, 1995, 1, 165.

34 N. Setoyama, K. Kaneko and F. Rodriguez-Reinoso, J. Phys. Chem., 1996, 100, 10331.

35 A. W. Thornton, A. Ahmed, S. K. Kannam, B. Todd, M. Majumder and A. J. Hill, J. Membr. Sci., 2015, 485, 1.

36 C. P. Lusher, M. F. Secca and M. G. Richards, J. Low Temp. Phys., 1988, 72, 71. 